\title{
Static quark-antiquark potential: Scaling behavior and finite-size effects in SU(3) lattice gauge theory
}

\author{
G. S. Bali and K. Schilling \\ Physics Department, Bergische Universität, Gesamthochschule Wuppertal, Gauss Strasse 20, 5600 Wuppertal, Germany
}

(Received 27 February 1992)

\begin{abstract}
We present results on the static $q \bar{q}$ potential from high-statistics simulations on $16^{4}, 24^{3} \times 32$, and $32^{4}$ lattices, using the standard Wilson action at $\beta=6.0,6.2$, and 6.4 on the Connection Machine CM2. To decrease noise and increase accuracy, we applied a suitable local smoothing technique on the spatial parts of Wilson loop operators. As a result, we find a violation of asymptotic scaling of the string tension, as signaled by $\sqrt{\sigma} / \Lambda_{L}=96.7(1.6)(2.6), 86.4(1.0)(1.9), 82.3(0.8)(1.7)$, for the three $\beta$ values, with statistical and systematic errors. We observe a linear confining potential up to distances of $2 \mathrm{fm}$. A volume of $(1.5 \mathrm{fm})^{3}$ appears to be sufficient to avoid finite-size effects within our statistical accuracy $(\approx 1 \%)$.
\end{abstract}

PACS number(s): 11.15.Ha, 12.38.Aw, 12.38.Gc

\section{INTRODUCTION}

Much effort has been spent recently to push lattice simulations of pure $\mathrm{SU}(2)$ gauge theory towards the continuum limit by increasing lattice sizes and $\beta$ values to the record $48^{3} \times 56$ and 2.85 , respectively [1]. Indeed, it appears worthwhile to focus part of the present-day computing power of parallel supercomputers, and the computing techniques of today, on the verification of asymptotic scaling, one of the old issues that has been with us ever since the pioneering paper of Creutz on the SU(2) confining potential back in 1979 [2].

In the present work we extend the theme to pure $\mathrm{SU}(3)$ gauge theory, to be more realistic with respect to QCD. One of our goals is to increase the statistical accuracy of string tension measurements to the $1 \%$ level in the region $6.0 \leq \beta \leq 6.4$, on appropriate lattices. This precision sets the scale for the systematic effects as well, which is a rather demanding goal.

Sooner or later, critical slowing down and increasing lattice volumes will bar the view on long-distance physics. So far it has been widely believed that asymptotic scaling sets in soon after $\beta=6.2$. We find that this is not yet the case up to $\beta=6.4$.

The work was done as a "warmup" for our local Con- nection Machine, which has sufficient memory (256 MByte) to handle $32^{4}$ lattices. The CM slicewise FORTRAN compiler produces fast code; we reach 600 MFlops for SU(3) matrix multiplications, and update times/link of $22(13) \mu \mathrm{sec}$ for the 10-hit Metropolis (overrelaxation [3]) algorithm on our 8K-CM2 system. Its programing environment enables us to carry out the entire computer experiment on one and the same machine.

In order to test the scaling behavior of $\mathrm{SU}(3)$ gauge theory, both the lattice spacing $a$ and the lattice volume $L_{S}^{3} \times L_{T}$ are varied over suitable range ( $T$ stands for the time direction as well as for temporal separations). The key parameters of our various computer runs are collected in Table I. This table contains, in addition, for ease of orientation, the lattice resolutions and physical volumes, as they emanated from the present investigations.

\section{METHODS}

\section{A. Smoothing operators}

Lattice gauge theory is known to be hampered by the fact that physical quantities of interest such as masses, potentials, and matrix elements are related to asymptotic properties of exponentially decreasing correlation functions in Euclidean time, and therefore prone to be

TABLE I. The simulated lattices. Physical units correspond to the choice $\sqrt{\sigma}=420 \mathrm{MeV}$ for the string tension. Errors include statistical and systematic effects.

\begin{tabular}{lccccc}
\hline \hline & \multicolumn{2}{c}{$\beta=6.0$} & \multicolumn{1}{c}{$\beta=6.2$} & \multicolumn{2}{c}{$\beta=6.4$} \\
\hline$L_{S}^{3} \times L_{T}$ & $16^{4}$ & $32^{4}$ & $24^{3} \times 32$ & $24^{3} \times 32$ & $32^{4}$ \\
$a / \mathrm{fm}$ & \multicolumn{2}{c}{$0.106(5)$} & $0.0759(22)$ & \multicolumn{2}{c}{$0.0577(18)$} \\
$a^{-1} / \mathrm{GeV}$ & \multicolumn{2}{c}{$1.85(8)$} & $2.60(7)$ & $3.42(10)$ \\
$V^{1 / 3}=a L_{S} / \mathrm{fm}$ & $1.70(7)$ & $3.41(15)$ & $1.82(5)$ & $1.38(4)$ & $1.85(6)$ \\
$T=\left(a L_{T}\right)^{-1} / \mathrm{MeV}$ & $116(3)$ & $57(3)$ & $81(2)$ & \multicolumn{2}{c}{$107(3)$} \\
Total No. of sweeps & 36000 & 6100 & 17000 & 22000 & 9000 \\
Thermalization phase & 1000 & 1000 & 1000 & 2000 & 2500 \\
No. of measurements & 140 & 102 & 260 & 200 & 65 \\
Off-axis measurements & Yes & Yes & No & No & Yes \\
\hline \hline
\end{tabular}


drowned in noise. Various improvement techniques have been invented in the past, such as smoothing local (i.e. link-related) fluctuations in observables or applying variational methods in order to achieve early asymptotics (in $T$ ) of the transfer matrix.

We start from the relation between Wilson loops, $W(R, T)$, and the potential $V(R)$,

$$
W(R, T)=C(R) e^{-T V(R)}\left[+C^{\prime}(R) e^{-T V^{\prime}(R)}\right],
$$

written in terms of its leading asymptotic and subleading contributions. We call the former the "ground-state" and the latter "excited-state" contribution. The aim of optimization strategies is to enhance, for each value of $R$, the weight of the ground-state term $C(R)$. A variational technique has been applied to this end by the UKQCD Collaboration in their recent high-statistics SU(2) project [1]. These authors use a variety of fat spatial links (generated by up to 110 APE recursive blocking iterations [4] with the coefficient of the straight link set to two). They observe an early ground-state dominance and extract the potential from ratios of superimposed fuzzy loops up to large spatial separations $R$, using exclusively low- $T$ data.

In this paper, we follow a strategy which is complementary to the UKQCD approach in the sense that we concentrate on reducing the ultraviolet noise rather than smearing the operators over all length scales. ${ }^{1}$ In this spirit, we attempt to improve the situation by smoothing spatial links via a local procedure: consider a spatial link variable $U_{i}(n)$ and the sum $\Pi_{i}(n)$ of the four spatial staples connected to it:

$$
\Pi_{i}(n)=\sum_{\substack{j=1, \ldots, 3 \\ j \neq i}} U_{j}(n+\hat{i}) U_{i}^{\dagger}(n+\hat{j}) U_{j}^{\dagger}(n) .
$$

We apply a gauge-covariant, iterative smoothing algorithm which replaces (in the same order as the Metropolis update, but within the measuring process) $U_{i}(n)$ $\rightarrow U_{i}^{\prime}(n)$ with the requirement that the local spatial action $S_{i}(n)=-\operatorname{Re} \operatorname{Tr}\left\{U_{i}(n) \Pi_{i}(n)\right\}$ is minimized. Contributions from excited states become more and more suppressed as we repeat this procedure. At $\beta=6.0$ (6.4), this reduction reaches saturation after 8-12 (25-30) such smoothing steps. The degree of the final ground-state dominance can be quoted in terms of the overlap $C(R)$. We reach values of 95 (80)\% for small (large) spatial separations $R$.

A technicality: for an individual smoothing step, as for the overrelaxation algorithm, one has to determine the $\mathrm{SU}(3)$ matrix $U$ that maximizes the expression $\operatorname{Re} \operatorname{Tr}\{U F\}$, with a given complex $3 \times 3$ matrix $F$. In order to satisfy this task, we apply a cyclic iterative scheme in the spirit of the Cabibbo-Marinari algorithm [7]. Each iteration consists of two steps: (1) Compute $V_{i}$ $(i=1,2,3)$ from the three diagonal $\mathrm{SU}(2)$ subgroups of $\mathrm{SU}(3)$, that maximize, in their order of occur-

\footnotetext{
${ }^{1}$ Our algorithm is reminiscent of lattice cooling techniques of previous authors $[5,6]$, yet it is different since we are "cooling" only inside time slices.
}

rence, $\operatorname{Re} \operatorname{Tr}\left\{V_{1}\left(U_{n} F\right)\right\}, \quad \operatorname{Re} \operatorname{Tr}\left\{V_{2}\left(V_{1} U_{n} F\right)\right\}, \quad$ and $\operatorname{Re} \operatorname{Tr}\left\{V_{3}\left(V_{2} V_{1} U_{n} F\right)\right\} ;$ (2) replace $U_{n+1}=V_{3} V_{2} V_{1} U_{n}$. The initial value $U_{0}$ is computed by Gram-Schmidt orthonormalization of $F^{\dagger}$. After three such iterations this method is found to yield the exact value of $U_{\max }$, within numerical accuracy.

\section{B. Sampling}

In order to maintain an appropriate stochastic movement of the gauge system through phase space with increasing $\beta$, we have combined one 10-hit Metropolis sweep with four successive overrelaxation sweeps. The algorithms were tuned to achieve $99.5 \%$ acceptance for an overrelaxation update and $50 \%$ acceptance for each Metropolis hit. Measurements were started after 1000-2500 thermalization sweeps.

For a proper sampling we want to keep control of autocorrelations within the computer time series. Autocorrelation times are extremely hard to determine. For our purposes, we estimate "autocorrelation times" $\tau$ by three different methods: (1) decay rates from exponential fits to the autocorrelation function, called $\tau_{\text {fit }}$; (2) timeintegrated autocorrelations, labeled $\tau_{\text {int }}$; (3) inversion of the relation

$$
\sigma^{2} \approx \sigma_{0}^{2} \operatorname{coth}\left(\frac{1}{2 \tau_{\sigma}}\right)
$$

between the variance of an observable and its autocorrelation time, denoted $\tau_{\sigma} . \sigma^{2}$ can be obtained by the blocking procedure described in Appendix A.

We display some of these characteristic quantities in Table II. The errors quoted for $\tau_{\text {int }}$ are obtained by binning the data sets into 10 subsamples and computing their variance. The values we find for $\tau_{\text {fit }}$ reflect that a direct measurement of the asymptotic decay rates is overly difficult within our statistics (1600-3500 measurements). Nevertheless we can conclude from the numbers in Table II that Wilson loops are only weakly correlated when separated by about 100 sweeps. ${ }^{2}$ We chose to perform measurements of smoothed Wilson loops about every 100 sweeps as a reasonable balance between updating and observation.

\section{Extraction of potential values}

The overlaps and potential values $C(R)$ and $V(R)$ are evaluated from the Wilson loop data according to Eq. (1). For very low- $T$ separations the data are contaminated by contributions from excited states. For this reason a low$T$ cut must be applied in the analysis to isolate the leading asymptotic term. High- $T$ values are prerequisite, however, to verify asymptotic behavior. This is particu-

\footnotetext{
${ }^{2}$ We verified, that the topological susceptibility needs an order of magnitude more effort to decorrelate at $\beta=6.4$.
} 
TABLE II. Estimates of "autocorrelation times" (in Monte Carlo sweeps). The values were measured on the $16^{4}$ and $24^{3} \times 32$ lattices every 10 sweeps. $\chi_{\text {top }}$ stands for the topological susceptibility (measured every 250,100 sweeps, respectively).

\begin{tabular}{ccccr}
\hline \hline Observable & Estimate & $\beta=6.0$ & $\beta=6.2$ & $\beta=6.4$ \\
\hline & $\tau_{\text {fit }}$ & $4.9 \pm 1.0$ & $2.3 \pm 1.2$ & \\
$W(2,2)$ & $\tau_{\text {int }}$ & $5.4 \pm 1.2$ & $15.2 \pm 3.8$ & \\
& $\tau_{\sigma}$ & 4.0 & 14.1 & 30.6 \\
& & & & \\
$W(4,2)$ & $\tau_{\text {fit }}$ & $3.2 \pm 0.5$ & $2.9 \pm 0.6$ & \\
& $\tau_{\text {int }}$ & $4.9 \pm 1.3$ & $6.2 \pm 1.2$ & \\
$W(4,4)$ & $\tau_{\sigma}$ & 3.3 & 50.5 & 67.0 \\
$W(6,6)$ & $\tau_{\sigma}$ & 2.4 & 30.1 & 29.9 \\
$W(8,8)$ & $\tau_{\sigma}$ & 2.6 & 14.5 & 62.5 \\
$\chi_{\text {top }}$ & $\tau_{\sigma}$ & & 35.5 & 28.6 \\
\hline \hline
\end{tabular}

larly important for the long-distance part of the potential, which goes along with a smaller gap between excited states and ground-state contributions [8]. We measure up to $T=10\left(T=8\right.$ on the $16^{4}$ lattice) and observe stability of results in the region $T \geq 3$. In order to be on the safe side of bias reduction, we only include data for $T \geq T_{\min }+1=4$ into our analysis. Measurements run over spatial distances up to $R=L_{S} / 2$ for the on-axis case and up to $R=\sqrt{2} L_{S} / 2$ and $R=\sqrt{3} L_{S} / 2$ (in two and three spatial dimensions, respectively) for the off-axis [9] geometries.
Since we aim at a $1 \%$ statistical error on the string tension, a careful analysis both of statistical and systematic effects is required. A statistical error estimate must incorporate correlation effects within the Monte Carlo times series of configurations and between measurements of different operators on individual configurations. Systematic effects originate from the lattice geometry (finite volume and finite $a$ ) as well as from biases introduced by the fitting procedures. In the present investigation we make a particular effort to deal with all these error sources.

We vary the volume, at fixed $a$, by a factor 2 . Finite- $a$ effects are estimated from off-axis potentials as well as by variation of $\beta$. The elimination of possible systematic errors due to $R$ and $T$ cuts and our statistical error analysis methods are discussed in Appendix A.

\section{STRING TENSION RESULTS}

Results are presented from five different lattices with linear spatial extent between 1.4 and $3.4 \mathrm{fm}$ and lattice spacings $a$ ranging from 0.058 to $0.106 \mathrm{fm}$. As one can see from Table I, three of our lattices are of nearly equal physical size, but different resolution. The off-axis measurements have been carried out on all $16^{4}$ and $32^{4}$ lattices.

Finite volume effects. We look for finite volume effects at $\beta=6.0$, where we dispose, with the $32^{4}$ and $16^{4}$ lattices, of the largest lever arm in volume variation. The sensitivity of the potential towards this variation is visualized in Fig. 1. To enhance details, the relative deviations between the two data sets and a given reference curve (fitted to $32^{4}$ data) are plotted in Fig. 1. There ap-

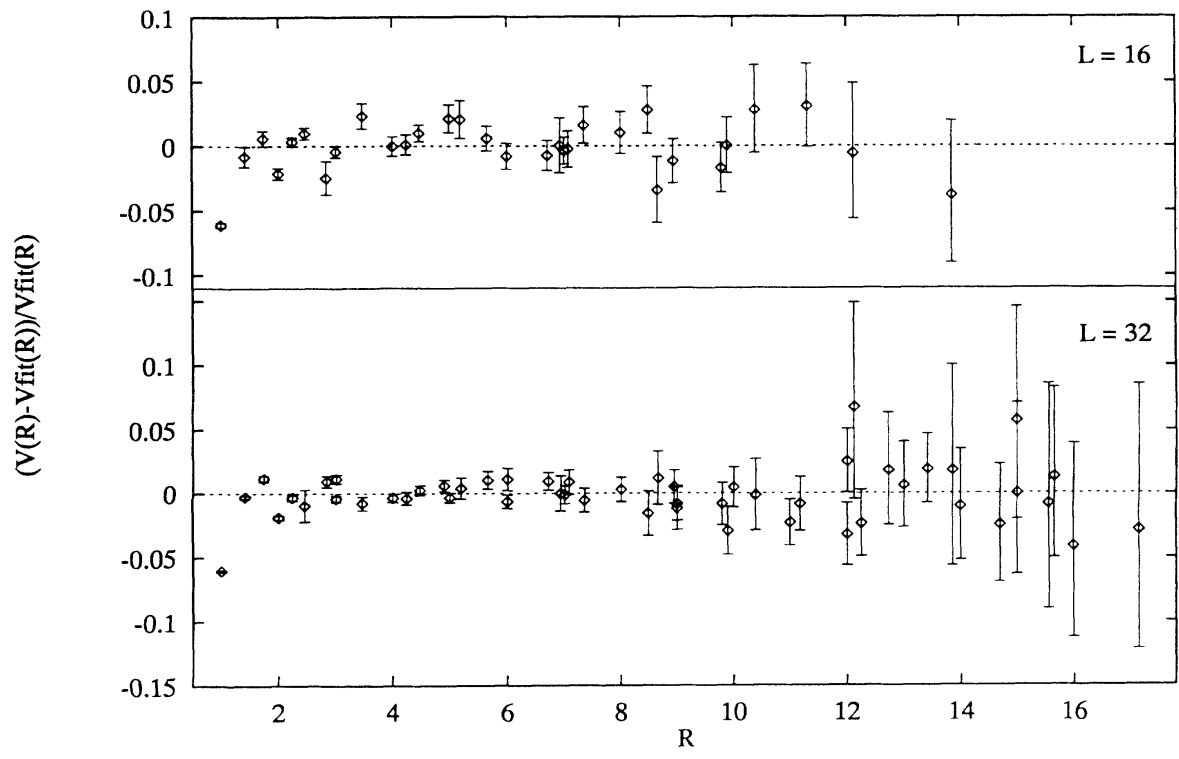

FIG. 1. The relative deviation between the potential values and the corresponding fit curve (taken from a fit to the $32^{4}$ data) is shown for the $32^{4}$ and $16^{4}$ lattices, respectively. 


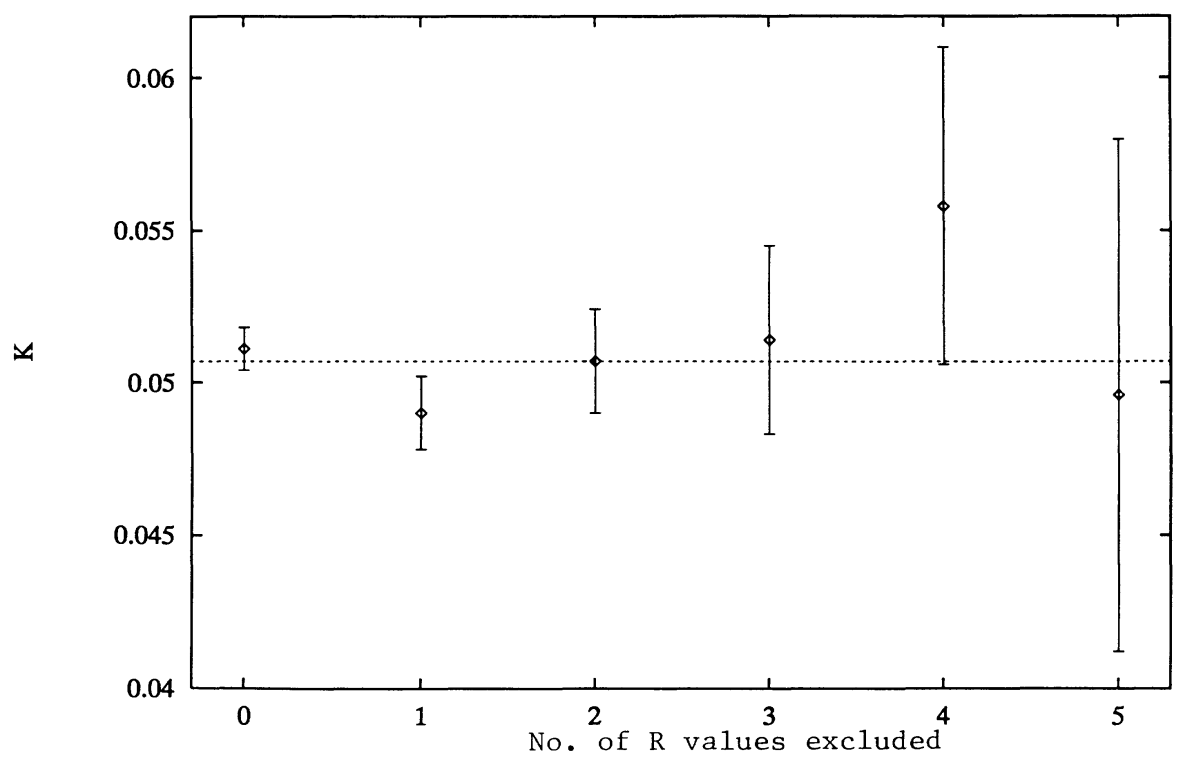

FIG. 2. The corresponding value for the string tension $K$ for the $32^{4}$ lattice at $\beta=6.0$ is plotted against the number of small $R$ values excluded in the fitting procedure. The quality of each fit $\left(\chi^{2} / N_{\mathrm{DF}}\right)$ is indicated next to the data point. The first two values have unreliable error bars since $\chi^{2} / N_{\mathrm{DF}}>1$.

pears to be no systematic difference in the potential, ${ }^{3}$ as we vary the lattice extent from 1.7 to $3.4 \mathrm{fm}$. We note in passing that in the region $R<2 \sqrt{2}$ the data sets exhibit identical incompatibility with the reference curve, i.e., with the underlying parametrization. This is due to strong lattice artifacts. Similar observations pertain to the comparison of potentials from the $24^{3} \times 32$ and $32^{4}$ lattices at $\beta=6.4$ : for the string tension we find no indication of systematic volume effects.

Coulomb strength and string tension. The potential is fitted to the rotationally invariant ansatz

$$
V(\mathbf{R})=V_{0}+K R-e / R \text {. }
$$

$R$ denotes the Euclidean distance between the quark and antiquark. In the instance of our off-axis measurements, the allowed $\mathbf{R}$ vectors are multiples of $(1,1,0),(2,1,0)$, $(1,1,1),(2,1,1)$, and $(2,2,1)$.

The above parametrization does not incorporate the lattice geometry. Lattice corrections become important for small $R$, and therefore we are forced to exclude some of these values from the fit. We demonstrate the impact of such cuts on the resulting value of the string tension $K$ for $\beta=6.0$ in Fig. 2. The small- $R$ data induces poor $\chi^{2}$ fits and instability of results. The fit parameters become stable as soon as the data points responsible for large $\chi^{2}$ are removed. For the following analysis we apply the minimal cut $R \geq 2 \sqrt{2}$.

Our fitting procedure and error analysis implies four steps that take into account possible systematic errors

\footnotetext{
${ }^{3}$ We point out that the larger error bars for the $16^{4}$ lattice are due to the smaller available $T$ range $(T \leq 8)$.
}

due to $R$ and $T$ cuts as well as $R-T$ correlations and autocorrelations within the Monte Carlo times series. The details are elaborated in Appendix A.

In Table III, we list the fitted values for $V_{0}, e$, and $K$ from the on-axis data (on five lattices) as well as the weighted average obtained on the six (one on- and five off-axis) parameter sets (on three lattices). The scatter of the fit parameters is indicative for the remaining violation of rotational symmetry on our lattices. Therefore, we will use their variance to estimate the systematic errors of our results.

We find the following values for the string tension $K(\beta)=\sigma a^{2}(\beta)$, obtained from the on-axis potentials:

$$
\begin{aligned}
& K(6.0)=0.0511(17), \quad K(6.2)=0.0262(6), \\
& K(6.4)=0.0147(3) .
\end{aligned}
$$

These values change little if the off-axis data are incorporated in the described fashion:

$$
\begin{aligned}
& K(6.0)=0.0515(17)(28), \\
& K(6.2)=0.0262(6)(10), \\
& K(6.4)=0.0151(3)(5) .
\end{aligned}
$$

The first error is the smallest statistical error of the different parameters ( 1 on and 5 off axis); the second one is the scatter, called the systematic error. ${ }^{4}$ Note that the Coulomb term tends to increase with $\beta$, but remains close to $\pi / 12$. The perimeter term $V_{0}$, on the other hand, appears to be fairly independent of $\beta$.

\footnotetext{
${ }^{4}$ At $\beta=6.2$ we estimate the systematic error by interpolation.
} 
TABLE III. Fit results. The average is taken over all on- and off-axis fits to the data. The error of the average is the weighted variance of the fit parameters. The error for the on-axis parameters is the statistical error.

\begin{tabular}{|c|c|c|c|c|c|c|c|c|}
\hline & \multicolumn{4}{|c|}{$\beta=6.0$} & \multirow{2}{*}{$\begin{array}{c}\beta=6.2 \\
24^{3} \times 32\end{array}$} & \multicolumn{3}{|c|}{$\beta=6.4$} \\
\hline Vol. & \multicolumn{2}{|c|}{$16^{4}$} & \multicolumn{2}{|c|}{$32^{4}$} & & $24^{3} \times 32$ & \multicolumn{2}{|c|}{$32^{4}$} \\
\hline Axis & On axis & Average & On axis & Average & On axis & On axis & On axis & Average \\
\hline$K$ & $0.0534(18)$ & $0.0515(34)$ & $0.0511(17)$ & $0.0515(28)$ & $0.0262(6)$ & $0.0145(4)$ & $0.0147(3)$ & $0.0151(5)$ \\
\hline$e$ & $0.267(6)$ & $0.254(10)$ & $0.278(31)$ & $0.240(36)$ & $0.303(8)$ & $0.303(6)$ & $0.297(4)$ & $0.277(28)$ \\
\hline$V_{0}$ & $0.625(8)$ & $0.632(23)$ & $0.635(15)$ & $0.626(21)$ & $0.635(5)$ & $0.610(4)$ & $0.608(2)$ & $0.603(7)$ \\
\hline$\chi / N_{\mathrm{DF}}$ & 0.99 & & 0.77 & & 0.84 & 0.94 & 0.92 & \\
\hline
\end{tabular}

In Fig. 3, we illustrate the quality of our data and of the potential fits with the example of the $32^{4}$ lattice at $\beta=6.4$. The various off-axis entries are indicated by different symbols. The error bars refer to statistical errors only, while the dashed error band incorporates both statistical and systematic errors (added linearly). For convenience, we include tables of all potential values $V(R)$ and overlaps $C(R)$ in Appendix B.

We find very good scaling of the potential data within our $\beta$ region. In Fig. 4 , the data are scaled to a universal curve by subtracting $V_{0}$ and measuring energies and distances in appropriate units of $\sqrt{K}$. The dashed curve corresponds to $R-\pi / 12 R$. If we inject for the string tension the value $\sqrt{\sigma} \approx 420 \mathrm{MeV}$, we gain the physical scales for $R$ and $V$ : note that we reach an horizon of 2 fm.

\section{DISCUSSION AND OUTLOOK}

Asymptotic scaling of the string tension is tested by the dimensionless quantity $c=\sqrt{\sigma} / \Lambda_{L}$, where we use the standard two-loop expansion for $a(\beta) \Lambda_{L}$. In Fig. 5 our results for the on-axis string tension are shown together with previous high-statistics results from the $M T_{C}$ Collaboration [10] and Ref. [11]. The error bars to our points refer to statistical errors from the full correlation analysis as elaborated in Appendix A. Since previous authors have been less stringent about correlations, we refrain from quoting their error estimates.

We find that asymptotic scaling is not reached up to $\beta=6.4$, but there is a definite flattening out of the $\beta$ dependence. Under the assumption that the string tension approaches the asymptotic scaling region from above we might convert our last data point at $\beta=6.4$ into a lower bound for $\Lambda_{L}$, which turns out to be $\Lambda_{L} \geq 5.10(.05)(.28) \mathrm{MeV}$, where the systematic error reflects both violation of rotational invariance and the uncertainty of the experimental string tension. This can be translated into other renormalization schemes such as the modified minimal subtraction ( $\overline{\mathrm{MS}}$ ) scheme in the four-flavor sector [12]:

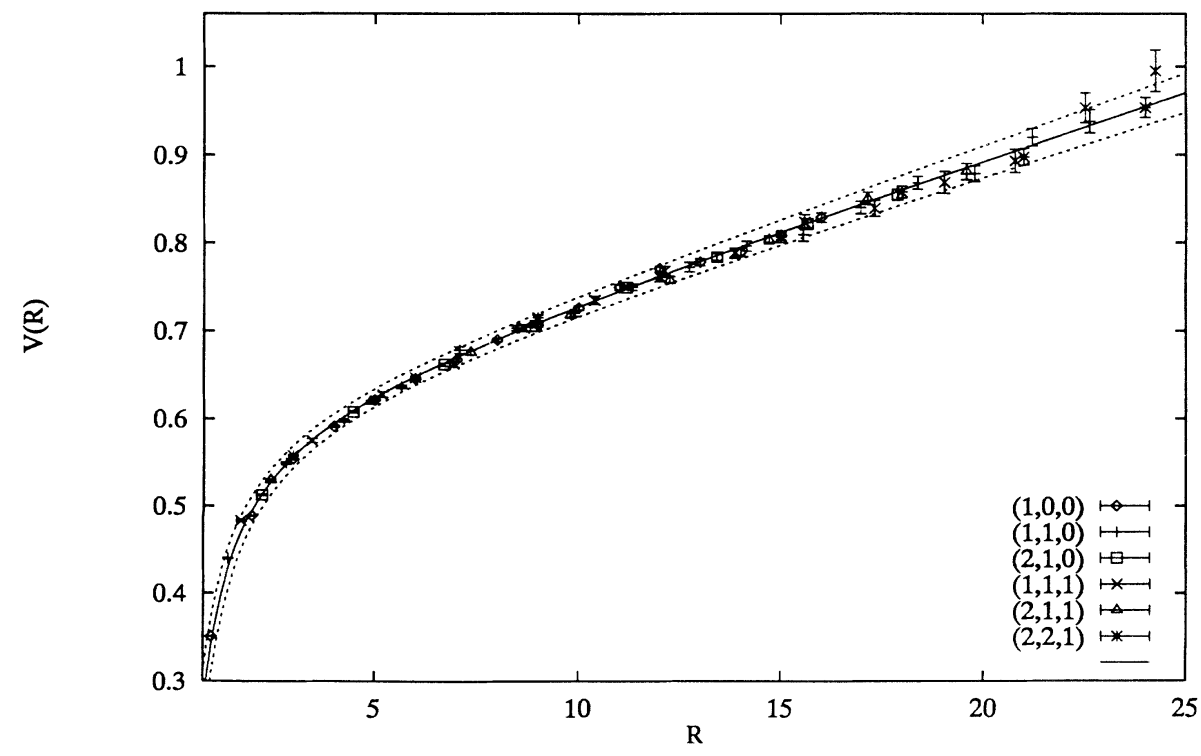

FIG. 3. The potential $V(R)$ for the $32^{4}$ lattice at $\beta=6.4$. The various off-axis entries are indicated by different symbols. The error bars refer to statistical errors only, while the dashed error band incorporates both statistical and systematic errors. 


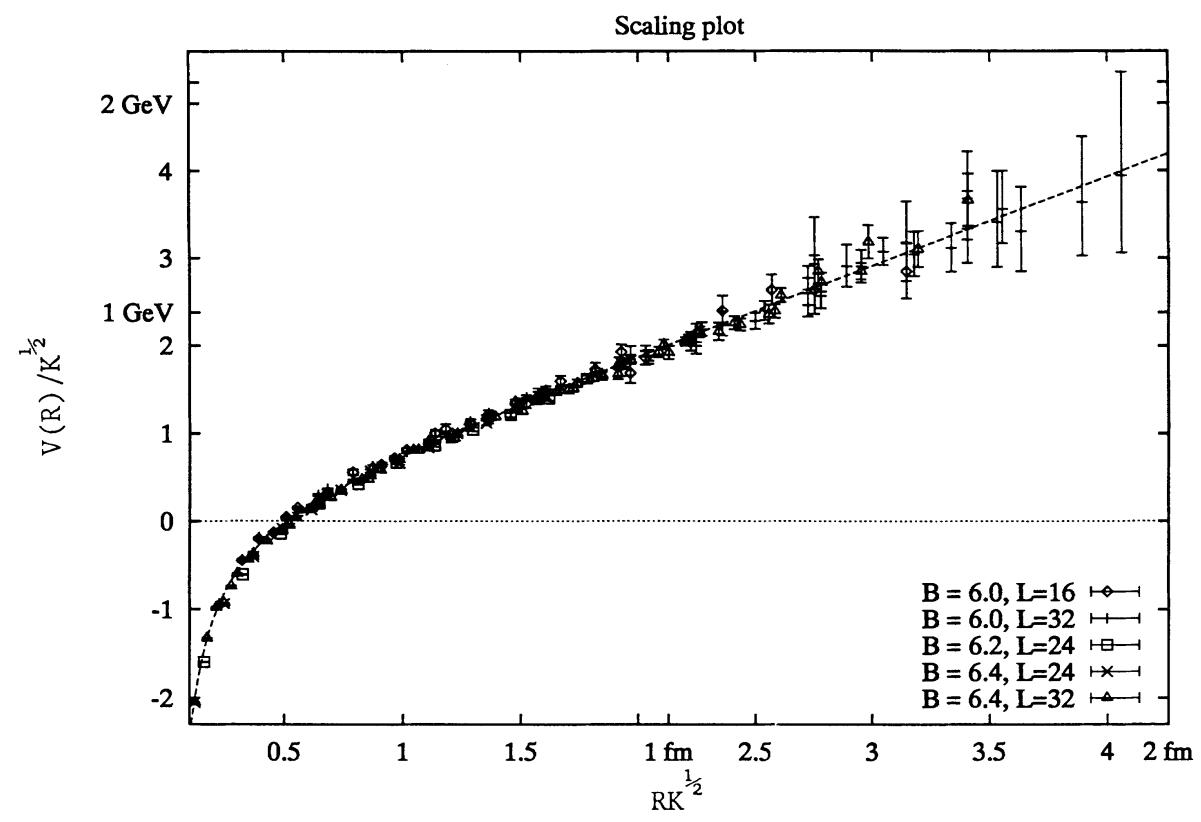

FIG. 4. All potential data of the five lattices have been scaled to a universal curve by subtracting $V_{0}$ and measuring energies and distances in appropriate units of $\sqrt{K}$. The dashed curve correspond to $V(R)=R-\pi / 12 R$. Physical units are calculated by exploiting the relation $\sqrt{\sigma} \approx 420 \mathrm{MeV}$.

$$
\Lambda \frac{(4)}{\mathrm{MS}}=46.1 \Lambda_{L} \geq 235(2)(13) \mathrm{MeV} \text {. }
$$

Needless to say, this value does not necessarily apply to full QCD.

In addition to the long-range behavior of the confining potential it is of considerable interest to investigate its ultraviolet structure. As we proceed into the weak coupling regime lattice simulations are expected to meet per- turbative results. Although we are aware that our lattice resolution is not yet really sufficient, we might dare to preview the continuum behavior of the Coulomb-like term from our results. In Fig. 6(a) [6(b)] we visualize the confidence regions in the $K-e$ plane from fits to various on- and off-axis potentials on the $32^{4}$ lattices at $\beta=6.0$ [6.4]. We observe that the impact of lattice discretization on $e$ decreases by a factor 2 , as we step up from $\beta=6.0$ to

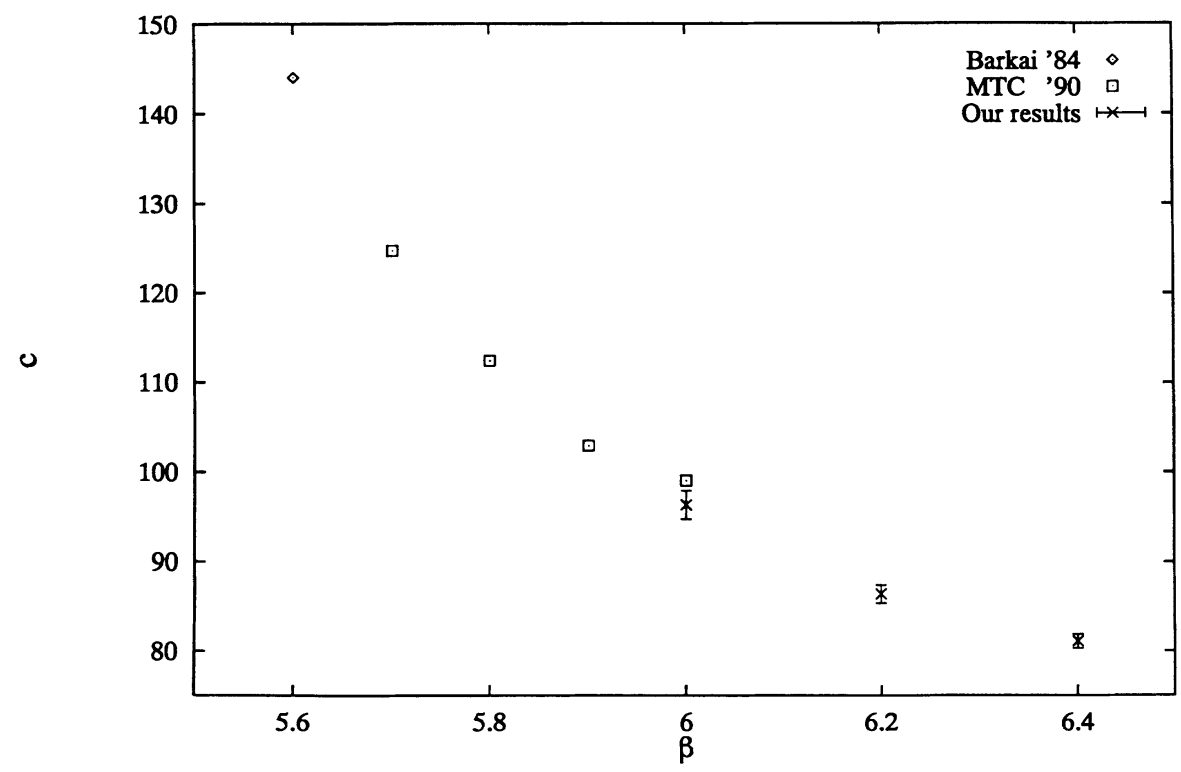

FIG. 5. The on-axis string tension [in units of the quantity $c=\sqrt{K} /\left(a \Lambda_{L}\right)$ ] as a function of $\beta$. Our results are combined with previous values obtained by the $M T_{C}$ collaboration [10] and Barkai, Moriarty, and Rebbi [11]. 

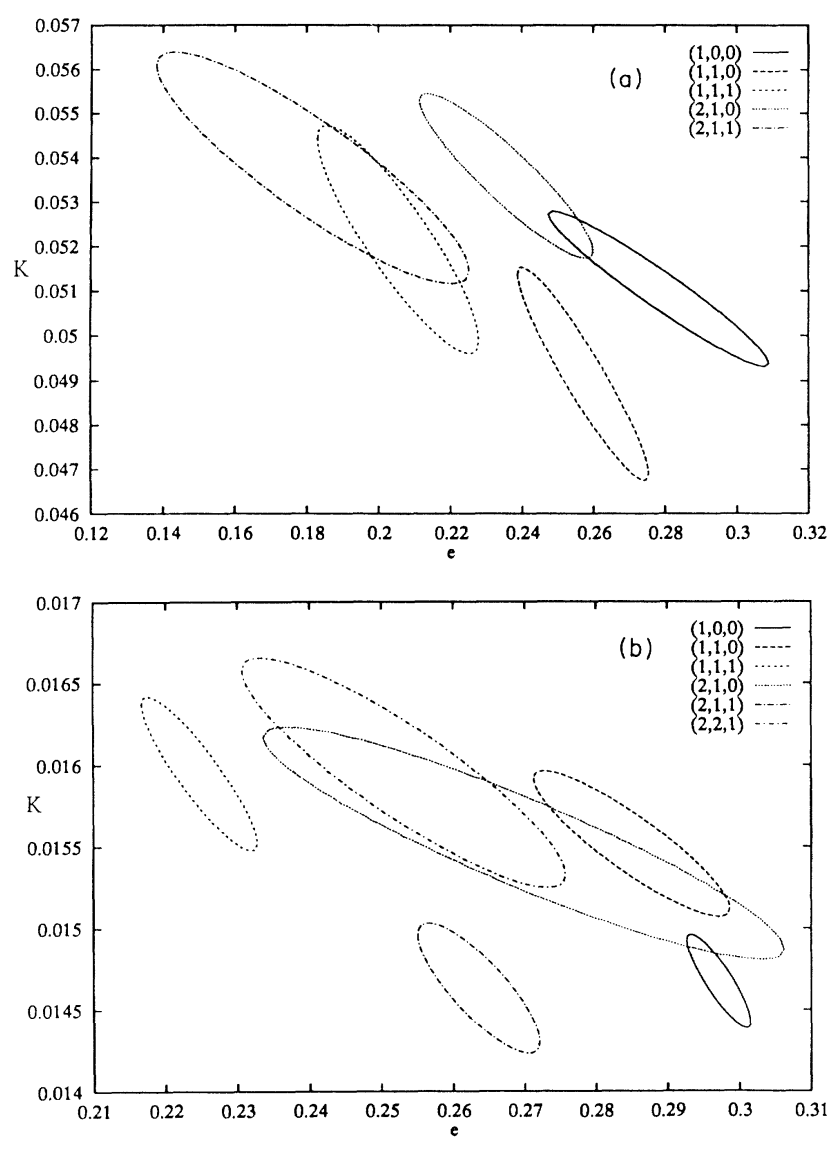

FIG. 6. Confidence regions $\left(\chi^{2}=\chi_{\min }^{2}+1\right)$ in the $K$-e plane are shown for various on- and off-axis potentials on the $32^{4}$ lattices at $\beta=6.0$ (a) and $\beta=6.4$ (b).

$\beta=6.4$ [note, that the scale of the $e$ axis is expanded by this factor between Figs. 6(a) and 6(b)]. Second, there is some weak indication that the coupling $e$ increases with $\beta$. It can be seen, that the coupling results still suffer from marked anisotropy effects, while the string tension as an infrared quantity behaves rather isotropic. It would be of interest to develop an improved analysis of the coupling by more sophisticated unfolding techniques of lattice effects with the help of perturbative methods, although the lattice resolution at $\beta=6.4$ still seems to be too coarse to allow for significant quantitative results.

\section{ACKNOWLEDGMENTS}

We are grateful to Deutsche Forschungsgemeinschaft for the support given to our CM2 project (Grant No. Schi 257/1-4). We thank Peer Ueberholz and the staff of Thinking Machines Corporation for their kind help.

\section{APPENDIX A: FITTING PROCEDURES AND ERROR ANALYSIS}

We determine the potential values $V(R)$, overlaps $C(R)$, and potential parameters $V_{0}, K$, and $e$ as well as their errors and $\chi^{2}$ 's from our smoothed, Wilson loop operators in four steps: First estimate correction factors to the naive errors in order to incorporate autocorrela- tions. Second decide on $T$ cuts. Third calculate $T$ correlations and determine operators with best ground state overlaps $C(R)$. Finally fit the Wilson loop data to the potential ansatz $V(R)=V_{0}+K R-e / R$ taking into account $R-T$ and autocorrelations.

Step 1: estimate autocorrelations. We estimate an observable by its average value $\langle W\rangle$ over a finite time series $W_{i}=W_{i}(R, T)$. The naive variance

$$
\left(\Delta W^{(1)}\right)^{2}=\frac{1}{N(N-1)} \sum_{i=1}^{N}\left(W_{i}-\langle W\rangle\right)^{2}
$$

will lead to an underestimated error, as a consequence of autocorrelations. The latter are taken into account by a straightforward blocking procedure. Consider

$$
\left(\Delta W^{(m)}\right)^{2}=\frac{1}{\frac{N}{m}\left(\frac{N}{m}-1\right)} \sum_{i=1}^{N / m}\left[\bar{W}_{i}^{(m)}-\langle W\rangle\right]^{2},
$$

where the data are partitioned into blocks of length $m$ and $\bar{W}_{i}^{(m)}$ is defined to be the average over the $i$ th block. We vary $m=1, \ldots, m_{\max }$ with $\tau \ll m_{\max } \ll N$ and conservatively correct the naive error estimate by the multiplicative factor

$$
f_{R T}=\max _{m}\left\{\Delta W^{(m)} / \Delta W^{(1)}\right\} .
$$

Step 2: choose $T$ cuts. Local masses are computed according to

$$
M(R, T)=-\ln \frac{\langle W(R, T+1)\rangle}{\langle W(R, T)\rangle} .
$$

For a given value of $R$, the $T$-cut $T_{\min }(R)$ is determined from the stability requirement that $M(R, T)$ is constant within errors over the region $T \geq T_{\min }(R)$. We only used $T \geq T_{\min }(R)+1$ to avoid a bias in the estimate of the results. The typical value for $T_{\min }$ came out to be three.

Step 3: $T$ correlations, optimized operators, and potential values. The determination of fit parameters to a correlated data set over $T$ requires knowledge of the covariance matrix of the uncorrelated time series:

$$
\begin{aligned}
\widetilde{C}_{T_{1} T_{2}}^{(R)}=\frac{1}{N(N-1)} \sum_{i=1}^{N}( & \left.W_{i}\left(R, T_{1}\right)-\left\langle W\left(R, T_{1}\right)\right\rangle\right) \\
& \times\left(W_{i}\left(R, T_{2}\right)-\left\langle W\left(R, T_{2}\right)\right\rangle\right) .
\end{aligned}
$$

As we have autocorrelations, this matrix is corrected by the ansatz

$$
C_{T_{1} T_{2}}^{(R)}=\widetilde{C}_{T_{1} T_{2}}^{(R)} f_{R T_{1}} f_{R T_{2}}
$$

The overlap and potential are fitted for each value of $R$ from the form

$$
w(T ; C(R), V(R))=C(R) \exp \{-T V(R)\}
$$

by minimizing 
TABLE IV. The potential values $V(R)$ (in lattice units $a^{-1}$ ), ground-state overlaps $C(R)$, number of smoothing steps $N_{\mathrm{opt}}$, and $\chi^{2} / N_{\mathrm{DF}}$ for $\beta=6.0\left(V=16^{4}, 32^{4}\right)$.

\begin{tabular}{|c|c|c|c|c|c|c|c|c|c|}
\hline \multirow[t]{2}{*}{$R$} & \multirow[t]{2}{*}{ Path } & \multicolumn{2}{|c|}{$V(R)$} & \multicolumn{2}{|c|}{$C(R)$} & \multicolumn{2}{|c|}{$N_{\text {opt }}$} & \multicolumn{2}{|c|}{$\chi^{2} / N_{\mathrm{DF}}$} \\
\hline & & $16^{4}$ & $32^{4}$ & $16^{4}$ & $32^{4}$ & $16^{4}$ & $32^{4}$ & $16^{4}$ & $32^{4}$ \\
\hline 1.00 & 1 & $0.4108(8)$ & $0.4111(3)$ & $0.936(2)$ & $0.936(1)$ & 4 & 3 & 0.84 & 0.58 \\
\hline 1.41 & 2 & $0.5249(41)$ & $0.5277(7)$ & $0.630(10)$ & $0.935(3)$ & 0 & 5 & 0.34 & 0.52 \\
\hline 1.73 & 4 & $0.5803(34)$ & $0.5836(14)$ & $0.908(9)$ & $0.918(5)$ & 6 & 4 & 0.51 & 0.99 \\
\hline 2.00 & 1 & $0.5962(27)$ & $0.5987(10)$ & $0.900(8)$ & $0.900(4)$ & 11 & 3 & 0.99 & 0.94 \\
\hline 2.24 & 3 & $0.6363(22)$ & $0.6320(14)$ & $0.917(6)$ & $0.902(5)$ & 6 & 5 & 0.51 & 0.48 \\
\hline 2.45 & 5 & $0.6608(30)$ & $0.6481(81)$ & $0.910(8)$ & $0.271(12)$ & 8 & 0 & 0.60 & 0.67 \\
\hline 2.83 & 2 & $0.6701(88)$ & $0.6934(30)$ & $0.805(27)$ & $0.752(9)$ & 17 & 1 & 0.97 & 0.85 \\
\hline 3.00 & 1 & $0.6978(32)$ & $0.6978(17)$ & $0.874(9)$ & $0.894(6)$ & 3 & 6 & 0.25 & 0.95 \\
\hline 3.00 & 6 & & $0.7087(23)$ & & $0.900(8)$ & & 8 & & 0.54 \\
\hline 3.46 & 4 & $0.7525(72)$ & $0.7296(40)$ & $0.910(20)$ & $0.841(13)$ & 9 & 8 & 0.46 & 0.32 \\
\hline 4.00 & 1 & $0.7722(58)$ & $0.7697(26)$ & $0.821(14)$ & $0.859(9)$ & 3 & 6 & 0.99 & 0.28 \\
\hline 4.24 & 2 & $0.7891(62)$ & $0.7852(39)$ & $0.889(16)$ & $0.873(13)$ & 10 & 9 & 0.31 & 0.68 \\
\hline 4.47 & 3 & $0.8109(52)$ & $0.8020(30)$ & $0.884(14)$ & $0.864(11)$ & 9 & 7 & 0.50 & 0.16 \\
\hline 4.90 & 5 & & $0.8347(38)$ & & $0.866(13)$ & & 9 & & 0.93 \\
\hline 5.00 & 1 & $0.8536(90)$ & $0.8331(31)$ & $0.741(15)$ & $0.861(11)$ & 3 & 7 & 0.88 & 0.31 \\
\hline 5.20 & 4 & $0.865(12)$ & $0.8511(70)$ & $0.894(33)$ & $0.877(25)$ & 6 & 9 & 0.26 & 0.32 \\
\hline 5.66 & 2 & $0.8805(83)$ & $0.8844(61)$ & $0.877(22)$ & $0.885(22)$ & 11 & 10 & 0.32 & 0.64 \\
\hline 6.00 & 1 & $0.8882(91)$ & $0.8895(45)$ & $0.822(23)$ & $0.834(16)$ & 6 & 9 & 0.27 & 0.69 \\
\hline 6.00 & 6 & & $0.9052(80)$ & & $0.875(27)$ & & 14 & & 0.99 \\
\hline 6.71 & 3 & $0.929(11)$ & $0.9449(66)$ & $0.846(29)$ & $0.891(24)$ & 12 & 9 & 0.83 & 0.69 \\
\hline 6.93 & 4 & $0.949(20)$ & $0.948(13)$ & $0.825(49)$ & $0.838(44)$ & 19 & 11 & 0.95 & 0.50 \\
\hline 7.00 & 1 & $0.9492(99)$ & $0.9515(63)$ & $0.862(26)$ & $0.862(23)$ & 11 & 9 & 0.54 & 0.59 \\
\hline 7.07 & 2 & $0.954(14)$ & $0.9650(92)$ & $0.868(35)$ & $0.905(34)$ & 11 & 10 & 0.62 & 0.80 \\
\hline 7.35 & 5 & $0.988(13)$ & $0.9668(91)$ & $0.895(37)$ & $0.835(30)$ & 15 & 12 & 0.44 & 0.87 \\
\hline 8.00 & 1 & $1.019(16)$ & $1.011(10)$ & $0.882(41)$ & $0.855(35)$ & 13 & 9 & 0.30 & 0.82 \\
\hline 8.49 & 2 & $1.064(19)$ & $1.019(18)$ & $0.934(56)$ & $0.797(56)$ & 13 & 10 & 0.72 & 0.81 \\
\hline 8.66 & 4 & $1.009(27)$ & $1.057(22)$ & $0.774(63)$ & $0.892(77)$ & 17 & 17 & 0.75 & 0.19 \\
\hline 8.94 & 3 & $1.048(18)$ & $1.066(14)$ & $0.816(44)$ & $0.844(46)$ & 10 & 8 & 0.98 & 0.33 \\
\hline 9.00 & 1 & & $1.054(13)$ & & $0.821(43)$ & & 9 & & 0.21 \\
\hline 9.00 & 6 & & $1.051(18)$ & & $0.794(57)$ & & 10 & & 0.29 \\
\hline 9.80 & 5 & $1.088(21)$ & $1.097(18)$ & $0.788(50)$ & $0.791(58)$ & 17 & 11 & 0.35 & 0.36 \\
\hline 9.90 & 2 & $1.112(24)$ & $1.079(21)$ & $0.860(64)$ & $0.763(65)$ & 11 & 14 & 0.62 & 0.88 \\
\hline 10.00 & 1 & & $1.123(14)$ & & $0.845(47)$ & & 9 & & 0.95 \\
\hline 10.39 & 4 & $1.170(39)$ & $1.137(32)$ & $0.92(10)$ & $0.84(11)$ & 17 & 16 & 0.98 & 0.70 \\
\hline 11.00 & 1 & & $1.144(22)$ & & $0.731(66)$ & & 8 & & 0.56 \\
\hline 11.18 & 3 & & $1.171(24)$ & & $0.817(81)$ & & 12 & & 0.97 \\
\hline 11.31 & 2 & $1.224(38)$ & & $0.95(11)$ & & 12 & & 0.87 & \\
\hline 12.00 & 1 & & $1.255(26)$ & & $0.94(10)$ & & 10 & & 0.998 \\
\hline 12.00 & 6 & & $1.185(30)$ & & $0.711(87)$ & & 12 & & 0.65 \\
\hline 12.12 & 4 & $1.224(65)$ & $1.314(95)$ & $0.83(17)$ & $0.82(33)$ & 9 & 4 & 0.79 & 0.84 \\
\hline 12.25 & 5 & & $1.208(32)$ & & $0.667(87)$ & & 7 & & 0.23 \\
\hline 12.73 & 2 & & $1.286(55)$ & & $0.92(21)$ & & 9 & & 0.86 \\
\hline 13.00 & 1 & & $1.285(48)$ & & $0.78(15)$ & & 7 & & 0.31 \\
\hline 13.42 & 3 & & $1.324(35)$ & & $0.88(12)$ & & 9 & & 0.54 \\
\hline 13.86 & 4 & $1.272(73)$ & $1.346(99)$ & $0.74(27)$ & $0.82(37)$ & 17 & 8 & 0.94 & 0.90 \\
\hline 14.00 & 1 & & $1.317(53)$ & & $0.80(17)$ & & 13 & & 0.64 \\
\hline 14.70 & 5 & & $1.333(63)$ & & $0.73(19)$ & & 12 & & 0.86 \\
\hline 15.00 & 1 & & $1.383(92)$ & & $0.62(23)$ & & 5 & & 0.90 \\
\hline 15.00 & 6 & & $1.46(11)$ & & $0.77(39)$ & & 5 & & 0.35 \\
\hline 15.56 & 2 & & $1.40(12)$ & & $0.81(46)$ & & 12 & & 0.39 \\
\hline 15.65 & 3 & & $1.435(94)$ & & $0.87(35)$ & & 20 & & 0.22 \\
\hline 16.00 & 1 & & $1.38(13)$ & & $0.44(24)$ & & 5 & & 0.62 \\
\hline 17.15 & 5 & & $1.45(16)$ & & $0.57(42)$ & & 8 & & 0.68 \\
\hline 17.89 & 3 & & $1.52(23)$ & & $0.59(41)$ & & 6 & & 0.87 \\
\hline
\end{tabular}


TABLE V. Same as Table IV for $\beta=6.2, V=24^{3} \times 32$.

\begin{tabular}{rccccc}
\hline \hline$R$ & Path & $V(R)$ & $C(R)$ & $N_{\text {opt }}$ & $\chi^{2} / N_{\text {DF }}$ \\
\hline 1.00 & 1 & $0.3770(2)$ & $0.941(1)$ & 4 & 0.39 \\
2.00 & 1 & $0.5366(9)$ & $0.613(2)$ & 0 & 0.38 \\
3.00 & 1 & $0.6118(12)$ & $0.893(4)$ & 4 & 0.26 \\
4.00 & 1 & $0.6671(19)$ & $0.766(5)$ & 2 & 0.48 \\
5.00 & 1 & $0.7038(16)$ & $0.891(6)$ & 10 & 0.55 \\
6.00 & 1 & $0.7431(22)$ & $0.880(8)$ & 10 & 0.91 \\
7.00 & 1 & $0.7748(25)$ & $0.872(9)$ & 10 & 0.30 \\
8.00 & 1 & $0.8044(35)$ & $0.842(12)$ & 10 & 0.60 \\
9.00 & 1 & $0.8312(41)$ & $0.824(13)$ & 10 & 0.85 \\
10.00 & 1 & $0.8614(49)$ & $0.801(15)$ & 10 & 0.42 \\
11.00 & 1 & $0.8974(61)$ & $0.815(19)$ & 10 & 0.36 \\
12.00 & 1 & $0.9260(59)$ & $0.788(18)$ & 10 & 0.89 \\
\hline \hline
\end{tabular}

$$
\chi_{(R)}^{2}=\sum_{T_{1}, T_{2} \geq T_{\min }(R)}\left(w\left(T_{1}\right)-\left\langle W\left(R, T_{1}\right)\right\rangle\right)\left(C^{(R)^{-1}}\right)_{T_{1} T_{2}}\left(w\left(T_{2}\right)-\left\langle W\left(R, T_{2}\right)\right\rangle\right) .
$$

Fitting is done after each smoothing step. Finally, for a given $R$, the optimal number of steps $N_{\text {opt }}$ is chosen to maximize the overlap $C(R)$ under the constraint of $\chi^{2} / N_{\mathrm{DF}} \leq 1$.

Step 4: $R$-T correlations, potential parameters, and $R$ cuts. For the final fit that takes into account all possible correlations between the data, we start from an estimate of the full correlated covariance matrix,

$$
C_{T_{1} T_{2}}^{R_{1} R_{2}}=\frac{f_{R_{1} T_{1}} f_{R_{2} T_{2}}}{N(N-1)} \sum_{i=1}^{N}\left(W_{i}\left(R_{1}, T_{1}\right)-\left\langle W\left(R_{1}, T_{1}\right)\right\rangle\right)\left(W_{i}\left(R_{2}, T_{2}\right)-\left\langle W\left(R_{2}, T_{2}\right)\right\rangle\right),
$$

and fit to the dependency

$$
\widetilde{w}\left(R, T ; C_{1}, \ldots, C_{R_{\max }}, V_{0}, K, e\right)=C_{R} \exp \left\{-\left(V_{0}+K R-e / R\right) T\right\}
$$

with different "overlap" parameters $C_{R}$ by minimizing

$$
\chi^{2}=\sum_{\substack{R_{1}, R_{2} \geq R_{\mathrm{cut}} \\ T_{1}, T_{2} \geq T_{\min }}}\left(\widetilde{w}\left(R_{1}, T_{1}\right)-\left\langle W\left(R_{1}, T_{1}\right)\right\rangle\right)\left(C^{-1}\right)_{T_{1} T_{2}}^{R_{1} R_{2}}\left(\widetilde{w}\left(R_{2}, T_{2}\right)-\left\langle W\left(R_{2}, T_{2}\right)\right\rangle\right) .
$$

Note that the inverse of the covariance matrix in each case is computed after application of the $T$ and $R$ cuts. Starting values of the parameters $V_{0}, K$, and $e$ are required for the minimization procedure that are taken from a "naive" fit of the potential data to the parametrization

$$
V(R)=V_{0}+K R-e / R \text {. }
$$

The initial values of $C_{R}$ are chosen from the $T$-correlation analysis [Eqs. (A7) and (A8)].

As a final result, we obtain $V_{0}, K$, and $e$ together with their errors, including all possible correlation effects. Stability of these parameters has been checked by discarding data for $R=1,2, \ldots$ successively.

TABLE VI. Same as Table IV for $\beta=6.4, V=24^{3} \times 32$.

\begin{tabular}{rccccc}
\hline \hline \multicolumn{1}{c}{$R$} & Path & $V(R)$ & $C(R)$ & $N_{\text {opt }}$ & $\chi^{2} / N_{\text {DF }}$ \\
\hline 1.00 & 1 & $0.3512(2)$ & $0.945(1)$ & 14 & 0.25 \\
2.00 & 1 & $0.4882(6)$ & $0.921(2)$ & 10 & 0.66 \\
3.00 & 1 & $0.5537(9)$ & $0.925(3)$ & 15 & 0.87 \\
4.00 & 1 & $0.5926(10)$ & $0.904(4)$ & 17 & 0.999 \\
5.00 & 1 & $0.6189(14)$ & $0.895(5)$ & 20 & 0.70 \\
6.00 & 1 & $0.6465(17)$ & $0.896(6)$ & 20 & 0.78 \\
7.00 & 1 & $0.6734(21)$ & $0.836(7)$ & 7 & 0.29 \\
8.00 & 1 & $0.6846(21)$ & $0.870(7)$ & 20 & 0.81 \\
9.00 & 1 & $0.7068(23)$ & $0.885(8)$ & 20 & 0.95 \\
10.00 & 1 & $0.7264(28)$ & $0.874(9)$ & 20 & 0.29 \\
11.00 & 1 & $0.7408(32)$ & $0.864(10)$ & 20 & 0.44 \\
12.00 & 1 & $0.7628(34)$ & $0.863(11)$ & 20 & 0.99 \\
\hline \hline
\end{tabular}


TABLE VII. Same as Table IV for $\beta=6.4, V=32^{4}$.

\begin{tabular}{|c|c|c|c|c|c|c|c|c|c|c|c|}
\hline$R$ & Path & $V(R)$ & $C(R)$ & $N_{\text {opt }}$ & $\chi^{2} / N_{\mathrm{DF}}$ & $R$ & Path & $V(R)$ & $C(R)$ & $N_{\text {opt }}$ & $\chi^{2} / N_{\mathrm{DF}}$ \\
\hline 1.00 & 1 & $0.3511(3)$ & $0.946(1)$ & 5 & 0.53 & 11.18 & 3 & $0.7490(25)$ & $0.899(9)$ & 30 & 0.89 \\
\hline 1.41 & 2 & $0.4400(6)$ & $0.945(2)$ & 9 & 0.33 & 11.31 & 2 & $0.7495(38)$ & $0.879(13)$ & 30 & 0.65 \\
\hline 1.73 & 4 & $0.4828(11)$ & $0.500(2)$ & 0 & 0.55 & 12.00 & 1 & $0.7705(29)$ & $0.846(10)$ & 14 & 0.98 \\
\hline 2.00 & 1 & $0.4887(7)$ & $0.922(2)$ & 11 & 0.84 & 12.00 & 6 & $0.7606(45)$ & $0.871(15)$ & 30 & 0.44 \\
\hline 2.24 & 3 & $0.5127(8)$ & $0.927(3)$ & 13 & 0.74 & 12.12 & 4 & $0.7678(53)$ & $0.911(19)$ & 30 & 0.84 \\
\hline 2.45 & 5 & $0.5298(11)$ & $0.724(3)$ & 1 & 0.32 & 12.25 & 5 & $0.7573(41)$ & $0.858(14)$ & 30 & 0.74 \\
\hline 2.83 & 2 & $0.5493(14)$ & $0.918(5)$ & 16 & 0.26 & 12.73 & 2 & $0.7721(55)$ & $0.886(20)$ & 30 & 0.37 \\
\hline 3.00 & 1 & $0.5541(9)$ & $0.881(3)$ & 3 & 0.30 & 13.00 & 1 & $0.7778(41)$ & $0.883(14)$ & 30 & 0.27 \\
\hline 3.00 & 6 & $0.5581(10)$ & $0.919(3)$ & 17 & 0.81 & 13.42 & 3 & $0.7836(39)$ & $0.868(13)$ & 30 & 0.46 \\
\hline 3.46 & 4 & $0.5751(22)$ & $0.903(8)$ & 17 & 0.38 & 13.86 & 4 & $0.7880(57)$ & $0.853(20)$ & 30 & 0.73 \\
\hline 4.00 & 1 & $0.5910(12)$ & $0.901(4)$ & 18 & 0.37 & 14.00 & 1 & $0.7890(46)$ & $0.852(15)$ & 30 & 0.62 \\
\hline 4.24 & 2 & $0.5976(17)$ & $0.903(6)$ & 12 & 0.26 & 14.14 & 2 & $0.7961(56)$ & $0.868(18)$ & 30 & 0.88 \\
\hline 4.47 & 3 & $0.6076(13)$ & $0.901(5)$ & 23 & 0.86 & 14.70 & 5 & $0.8031(47)$ & $0.846(15)$ & 30 & 0.36 \\
\hline 4.90 & 5 & $0.6193(13)$ & $0.895(5)$ & 23 & 0.91 & 15.00 & 1 & $0.8084(52)$ & $0.861(17)$ & 30 & 0.53 \\
\hline 5.00 & 1 & $0.6211(12)$ & $0.907(4)$ & 23 & 0.44 & 15.00 & 6 & $0.8064(60)$ & $0.847(19)$ & 28 & 0.26 \\
\hline 5.20 & 4 & $0.6271(31)$ & $0.909(10)$ & 27 & 0.77 & 15.56 & 2 & $0.8091(72)$ & $0.843(23)$ & 30 & 0.50 \\
\hline 5.66 & 2 & $0.6364(21)$ & $0.888(7)$ & 24 & 0.35 & 15.59 & 4 & $0.8236(86)$ & $0.872(30)$ & 26 & 0.26 \\
\hline 6.00 & 1 & $0.6451(14)$ & $0.890(5)$ & 25 & 0.55 & 15.65 & 3 & $0.8216(49)$ & $0.869(16)$ & 30 & 0.34 \\
\hline 6.00 & 6 & $0.6464(21)$ & $0.880(7)$ & 14 & 0.98 & 16.00 & 1 & $0.8287(54)$ & $0.834(18)$ & 22 & 0.99 \\
\hline 6.71 & 3 & $0.6615(18)$ & $0.900(6)$ & 28 & 0.46 & 16.97 & 2 & $0.8399(72)$ & $0.836(23)$ & 30 & 0.79 \\
\hline 6.93 & 4 & $0.6621(35)$ & $0.871(11)$ & 24 & 0.50 & 17.15 & 5 & $0.8505(70)$ & $0.874(24)$ & 30 & 0.71 \\
\hline 7.00 & 1 & $0.6667(16)$ & $0.893(6)$ & 27 & 0.36 & 17.32 & 4 & $0.8391(93)$ & $0.789(29)$ & 23 & 0.25 \\
\hline 7.07 & 2 & $0.6782(39)$ & $0.589(9)$ & 2 & 0.83 & 17.89 & 3 & $0.8546(62)$ & $0.835(21)$ & 30 & 0.26 \\
\hline 7.35 & 5 & $0.6753(28)$ & $0.895(9)$ & 20 & 0.99 & 18.00 & 6 & $0.8578(68)$ & $0.818(22)$ & 25 & 0.998 \\
\hline 8.00 & 1 & $0.6895(21)$ & $0.894(7)$ & 30 & 0.42 & 18.39 & 2 & $0.8681(74)$ & $0.867(25)$ & 30 & 0.54 \\
\hline 8.49 & 2 & $0.7028(30)$ & $0.853(10)$ & 11 & 0.99 & 19.05 & 4 & $0.869(12)$ & $0.829(41)$ & 30 & 0.57 \\
\hline 8.66 & 4 & $0.7030(40)$ & $0.904(14)$ & 30 & 0.79 & 19.60 & 5 & $0.8811(93)$ & $0.815(29)$ & 30 & 0.55 \\
\hline 8.94 & 3 & $0.7054(20)$ & $0.881(7)$ & 30 & 0.72 & 19.80 & 2 & $0.8787(88)$ & $0.801(28)$ & 30 & 0.50 \\
\hline 9.00 & 1 & $0.7064(20)$ & $0.888(7)$ & 30 & 0.64 & 20.79 & 4 & $0.893(13)$ & $0.795(42)$ & 30 & 0.37 \\
\hline 9.00 & 6 & $0.7146(31)$ & $0.848(10)$ & 11 & 0.99 & 21.00 & 6 & $0.8974(94)$ & $0.798(29)$ & 30 & 0.33 \\
\hline 9.80 & 5 & $0.7174(33)$ & $0.860(11)$ & 25 & 0.26 & 21.21 & 2 & $0.9198(99)$ & $0.849(33)$ & 25 & 0.45 \\
\hline 9.90 & 2 & $0.7195(31)$ & $0.883(11)$ & 30 & 0.47 & 22.52 & 4 & $0.953(17)$ & $0.902(62)$ & 30 & 0.77 \\
\hline 10.00 & 1 & $0.7256(25)$ & $0.878(8)$ & 25 & 0.26 & 22.63 & 2 & $0.938(13)$ & $0.827(42)$ & 30 & 0.83 \\
\hline 10.39 & 4 & $0.7345(45)$ & $0.886(15)$ & 30 & 0.83 & 24.00 & 6 & $0.954(11)$ & $0.806(36)$ & 30 & 0.92 \\
\hline 11.00 & 1 & $0.7492(30)$ & $0.864(10)$ & 15 & 0.92 & 24.25 & 4 & $0.995(23)$ & $0.904(85)$ & 26 & 0.99 \\
\hline
\end{tabular}

\section{APPENDIX B: POTENTIAL VALUES}

We measured on-axis potentials as well as 5 different off-axis potentials constructed from Wilson loop operators with smoothed spatial insertions along the following paths:

$$
\text { Path No. }
$$

Path $(X, Y, Z)$

$(1,0,0)$

Elementary distance $D$
$(1,1,0)$

1

1.41

12

2

$1,0)$
3

$(2,1,0)$

2.24
4

$(1,1,1)$

1.73
5

$(2,1,1)$

2.45
6

$(2,2,1)$

. 
[1] UKQCD Collaboration, S. P. Booth, K. C. Bowler, D. S. Henty, R. D. Kenway, B. J. Pendleton, D. G. Richards, A. D. Simpson, A. C. Irving, A. McKerrell, C. Michael, P. W. Stephenson, M. Teper, and K. Decker, Phys. Lett. B 275, 424 (1992).

[2] M. Creutz, Phys. Rev. Lett. 43, 553 (1979); Phys. Rev. D 21, 2308 (1980).

[3] S. L. Adler, Phys. Rev. D 23, 2901 (1981).

[4] APE Collaboration, M. Albanese et al., Phys. Lett. B 192, 163 (1987).

[5] J. Hoek, M. Teper, and J. Waterhouse, Nucl. Phys. B288, 589 (1987).

[6] M. Campostrini, A. Di Giacomo, M. Maggiore, H. Panagopoulos, and E. Vicari, Phys. Lett. B 225, 403 (1989).
[7] N. Cabibbo and E. Marinari, Phys. Lett. 119B, 387 (1982).

[8] N. A. Campbell, A. Huntley, and C. Michael, Nucl. Phys. B306, 51 (1988); S. Perantonis, and C. Michael, ibid. B347, 854 (1990).

[9] C. B. Lang and C. Rebbi, Phys. Lett. 115B, 137 (1982).

[10] $M T_{C}$ Collaboration, K. D. Born et al., in Lattice ' 90 , Proceedings of the International Symposium, Tallahassee, Florida, 1990, edited by U. M. Heller, A. D. Kennedy, and S. Sanielevici [Nucl. Phys. B (Proc. Suppl.) 20, 394 (1991)].

[11] D. Barkai, K. J. M. Moriarty, and C. Rebbi, Phys. Rev. D 30, 1293 (1984).

[12] A. Hasenfratz and P. Hasenfratz, Phys. Lett. 93B, 165 (1980); W. Celmaster and R. J. Gonsalves, Phys. Rev. D 21, 3112 (1980). 